\title{
Off to new pastures: Exploring Tensions Between Followers and Leaders in the Automotive industry Challenging the Adoption of Digital Leadership
}

\author{
Julia K. Eberl \\ Leuphana University of Lüneburg \\ Julia.Eberl@stud.leuphana.de
}

\author{
Paul Drews \\ Leuphana University of Lüneburg \\ Paul.Drews@leuphana.de
}

\begin{abstract}
The automotive industry is driving digitalization at high speed to optimize operations, gain new business opportunities, and close the gap with new, leading competitors from the IT industry. Although the automotive industry aims for digital leadership (DL) to transfer from antecedent physical products to new pastures of digital services, the adoption is challenged by tensions in the follower-leader relationship (FLR). To identify these tensions for the first time in research, we analyzed $D L$ in the automotive industry from a follower and leader perspective. Based on 25 interviews, the results extend existing research on the adoption of $D L$ in the automotive industry by (a) identifying four configurations of digital leadership adoption stages causing tensions in the FLR which impede the adoption of $D L$ and (b) four strategies for managing the tensions.
\end{abstract}

\section{Introduction}

"We were like a [digital] virus that tried to infect a body that is drilled to produce premium cars, and the immune system destroyed us without mercy." (L5)

Although a successful defense against a virus is commonly known as the desirable reaction of an immune system, in this case, it is not. The automotive industry is dominated by companies that are optimized to produce physical products [1]. However, with the increasing customer demand for a shared, autonomous, and connected mobility experience, the current business model (BM) of the automotive industry is challenged by digital technologies [2] which require a more agile organizational environment [1]. The combination of these two portfolios, the physical product and the digital product, leads to tensions in the companies. Unfortunately, the legacy organization, as the stronger power, is often successful in defending the organization enabled for digital products [2].

Nonetheless, it is not impossible. Whereas automotive companies with a long history struggle with these tensions, new market players, such as Tesla, BYD, and NIO, show that the heritage of producing cars is no longer necessary to become a valuable, or even the most valuable, automotive company in the market. This high market capitalization is mainly the reward for the newcomers' innovative BMs [2]. To jump on that ride as well, Volkswagen CEO Herbert Diess announced his strategy to become a software company by 2025 [3] to keep pace in identifying new digital BMs. However, it is not only about using digital technology but also about adopting digital leadership (DL) to lead the company towards digital BMs [4].

Previous research on DL focused on the skills and role of the digital leader, as well as the organizational and individual levels of leadership [5]. However, DL research is still characterized by high fuzziness of the definition and determinants, as well as the adoption of DL. Moreover, except Bolte et al. [6], researchers have neglected to include followers' perspective in observations. Although Avolio et al. [7] emphasized the importance of followers' influence on leadership, research did not analyze the synergies and tensions in the follower-leader relationship (FLR) for DL.

The automotive industry is in the middle of adopting DL, however, it is not adopted consistently across different departments of an automotive company. Therefore, in this article, we leverage the adoption status of DL in the automotive industry to close two research gaps, (a) understanding and adoption of DL in the FLR and (b) strategies improving the adoption of DL, by answering the following research questions: What tensions between followers and leaders are impeding the adoption of DL in the automotive industry? What strategies exist to manage these tensions?

To answer these questions, we used grounded theory to analyze 25 interviews with experts in followership and leadership roles in the automotive industry. As the first contribution of this work, we clustered the FLR by the DL adoption stage (DLAS) and identified four constellations causing tensions in the adoption of DL by including the perspective of the 
followers as a main influence factor on leadership. Furthermore, we investigated the causes of the tensions and categorized them by the organizational tension framework of Smith and Lewis [8]. As a second contribution, we explain four strategies for managing the tensions in the FLR. The aim is to increase successful DL adoption by identifying and remediating tensions in the FLR.

The remainder of the paper is structured as follows. In section 2, we introduce the theoretical background of DL, the domain of the automotive industry as well as tensions. In section 3 , we describe the methodology; in section 4, we present the findings; and in section 5, we discuss the implications for leadership research and practitioners as well as limitations. Last, in section 6 , we summarize the conclusion and outlook.

\section{Conceptual foundations}

\subsection{Digital leadership}

Although DL research grew in the last 5 years, it is still characterized by fuzziness. A high variety of definitions of DL exists. Klus and Müller [9] used DL as a synonym for E-Leadership that describes leadership by using digital technology to influence behavior of followers [10]. However, various definitions of DL disprove the limitation of DL to support existing business with technology. El Sawy et al. [11] added the aspect of ensuring the success of the enterprise by including the business ecosystem in the digitalization. Therefore, we define digital leadership based on the three levels of DL, the leader, the organizational and the individual level, as "a complex construct aiming for a customer-centered, digitally enabled, leading-edge business model by (1) transforming the role, skills, and style of the digital leader, (2) realizing a digital organization, including governance, vision, values, structure, culture, and decision processes, and (3) adjusting people management, virtual teams, knowledge, and communication and collaboration on the individual level" [5].

First, DL focuses on the digital leader. Most authors investigated the skills and competencies of a digital leader, which is why DL is mainly allocated in traits theory. Thus, the digital leader is described as a visionary, an innovative thinker, collaborative, flexible, open, and digitally savvy [5]. In contrast to the skills of the digital leader, the leadership style is rarely discussed and classified in the context of neocharismatic leadership theories. Prince [12] identified aspects of transactional, transformational, and authentic leadership in DL.
Second, DL enables individuals for digitalization on the individual level. Resulting from the constant change of technology, digital leaders need to motivate people for continuous learning [13]. Moreover, the digital leader coaches followers in working together virtually across teams to flexibly bring together different disciplines and skills [13]. Therefore, DL drives collaboration activities [13].

Third, DL creates an organization driving digitalization on the organizational level. To bring forward digitally enabled BMs, the vision is at the center of DL. Following the vision, the digital leader enables a culture of experimentation, failure, and innovation [14]. In addition, the digital leader needs to ensure that decisions, processes, and organizational structures support the innovative culture [13].

\subsection{Automotive Industry}

Born in the industrial age, the automotive industry has proven its strength in the engineering discipline by producing high quality cars. However, triggered by the new competitors on the market and changed customer demands [15], automotive companies are forced to drive digital innovation and extend their BM which require a deviation from established competencies and organization. The physical products are developed and produced in a sequential process starting with an incremental design, a strict mass production system and quality controls [2]. In contrast, the digital innovation process is iterative, rapidly innovating, and service-oriented [1].

To enable a successful intersection of physical products and digital technology, Piccini at al. [1] recommend the adoption of DL to establish a start-up culture, agile methodologies and increase digital knowledge in the core of the company. Although literature describes M\&A and bottom-up driven subcommunities [15] as strategies to drive change, the automotive industry lacks openness of the legacy organization.

While tensions exist in the transition from physical to digital BMs, it is unclear how these tensions affect the followers and leaders in the adoption of DL. Moreover, the tensions were not analyzed with regards to the tension categories described in chapter 2.3. Hence, we need to investigate the adoption status to DL and identify tensions which are blocking a further growth of DL.

\subsection{Tensions and management strategies}

Organizational tensions were analyzed by Lewis [16] defining tensions as "cognitively or socially 
constructed polarities that mask the simultaneity of conflicting truths."

We categorized the inductively identified tensions of our theory by the following tension categories [8]: (a) learning tensions (LT) resulting from leveraging and destroying the past to drive change and innovation; (b) belonging tensions (BT) caused by competing values of the individual and the collective; (c) performing tensions (PT) that arise from multiple, competing objectives of stakeholders seeking conflicting organizational success; and (d) organizing tensions (OT) that occur in complex systems aiming for an objective by using competing processes. Last, tensions are surfaced in the interrelation (abbreviated as "::") of the four tension categories. For example, tensions between learning and belonging (LT::BT) occur when individuals identify a need to change that is interfering with the individuals belonging to a group not favoring the change.

\section{Research design}

\subsection{Method}

The study has the goal to shed light on the rarely investigated phenomenon of tensions in the FLR causing heterogenous success of DL adoption in the automotive industry and strategies for managing these tensions. Considering the limited research on DL adoption and the focus on quantitative methods for including the followers' perspective on DL, we decided to build on an exploratory, qualitative research. Moreover, our objective was to develop theory. Therefore, we chose Corbin and Strauss' grounded theory approach [17]. Therefore, we conducted semi-structured interviews with experts in different automotive companies. The experts with responsibility for employees are categorized as leaders (L1 to L14), others as followers (F1 to F10). All experts have a track record of digitalization projects. The semi-structured interviews were based on two interview guides adjusted to the follower and leader perspectives. We iteratively developed both guides after the first follower and first leader interviews. We modified the guides again after the first and second thirds of both groups. Because of the interview format, we were able to focus on specific directions of the research during the data collection process. To mitigate risks in the qualitative interviews, we followed Myers and Newmann's recommendations [18] such as considering everyone as an interpreter, the researcher's situation, and clarifying confidentiality.

The unit of analysis is the FLR influenced by the three levels of DL and their determinants which we identified in existing DL research: the leader, the individual level and the organizational level [5]. The leader is described by the skills, role and leadership style. The individual level includes the direct leader leading individual followers and the respective team. The individual level can be structured in the determinants people management, virtual teams, knowledge, communication, and collaboration. The organizational level is part of DL as it influences the FLR by the determinants culture, values, vision, governance, processes, and structure. As the goal is to understand tensions in adopting DL at all levels of DL, we structured the interview questions accordingly. First, interviewees described their position, experience, and their understanding of DL. Moreover, the interviewees explained the current DLAS of the company and aligned it to the existing stages and characteristics of the Adapt2Digital's digital business transformation maturity framework [19] as shown in Figure 2. We chose the framework of Adapt2Digital because of the focus on leadership determinants.

\begin{tabular}{|c|c|c|}
\hline & Characteristics & Adoption stages of DL \\
\hline 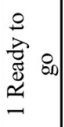 & $\begin{array}{l}\text { - Restrictive corporate } \\
\text { structure } \\
\text { - No adaptive culture } \\
\text { - Ad hoc digital skills }\end{array}$ & $\begin{array}{l}\text { Digitalization of the } \\
\text { existing processes without } \\
\text { changes }\end{array}$ \\
\hline 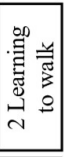 & $\begin{array}{ll}\text { - } & \text { Little integration } \\
\text { Emerging adaptive } \\
\text { culture } \\
\text { - } \\
\text { Digital has strategic } \\
\text { values }\end{array}$ & $\begin{array}{l}\text { Increasing growth of } \\
\text { bottom-up grass-route } \\
\text { movements but missing } \\
\text { adoption of the } \\
\text { organization }\end{array}$ \\
\hline 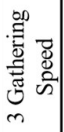 & $\begin{array}{l}\text { - Test \& Learn culture } \\
\text { Processes to enable } \\
\text { change to DL }\end{array}$ & $\begin{array}{l}\text { Driving innovation in } \\
\text { incubations, but no spill- } \\
\text { over to the core } \\
\text { organization }\end{array}$ \\
\hline 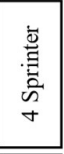 & $\begin{array}{ll}\text { - } & \text { Adaptive culture } \\
\text { - } & \text { Converged digital } \\
\text { processes } \\
\text { - } \\
\text { Deeper audience } \\
\text { connections } \\
\end{array}$ & $\begin{array}{l}\text { Organizational legitimacy } \\
\text { building for existing digital } \\
\text { business offerings }\end{array}$ \\
\hline 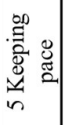 & $\begin{array}{l}\text { - Predictive scenarios } \\
\text { - Adaptive business } \\
\text { model } \\
\text { - Customer Centric }\end{array}$ & $\begin{array}{l}\text { High frequency in adjusting } \\
\text { organization to changed } \\
\text { conditions }\end{array}$ \\
\hline
\end{tabular}

Figure 1. Characteristics based on [19] and DLAS

Second, interviewees characterized the determinants with regards to the overall company and described changes in the last five years.

\subsection{Data}

We conducted 25 interviews between November 2020 and February 2021 via internet communication tools using audio and video transmission, except for 
two interviewees (L6 and L13) who used only audio. All interviews $(\mathrm{n}=25)$ were recorded via software $(\mathrm{n}$ $=24$ ) or manually $(\mathrm{n}=1)$, transcribed and coded by the authors. All interviews were scheduled for 60 minutes. One interviewee asked for the questions in advance and divided the interview into two scheduled meetings, 30 minutes each, with a break of 5 working days between the meetings.

The initial list of interviewees was generated by using our professional network $(\mathrm{n}=8)$ and searching for digitalization experts in automotive companies via Linked Sales Navigator $(n=17)$ and checking their fit based on their expert reputation. In this way, we selected interviewees with diverse genders, experience, regional perspectives, culture, and profession The interviewees have extensive experience in different positions in top, middle and low-level management in the automotive industry dealing with different aspects of digitalization from workers' council, marketing leads, head of transformation, subject matter experts in $R \& D$, Finance, and IT. The average years of experience in automotive industry is 13.4. We leveraged the interviewees' experience by asking more detailed questions according to their interviews. Moreover, the ongoing data analysis allowed us to select additional interview candidates to shed light on specific aspects. For example, after five interviews we identified a lack of perspective from mobility services, especially followers moving from car manufacturers to mobility services. Moreover, interviewees reported that colleagues were sent to Silicon Valley to experience digital leadership and bring it back to the corporation. Therefore, we included an interviewee who was part of the program.

The above-mentioned considerations led us to the list of interviewees (I) categorized as followers (F) and leaders (L), including the department, management level (ML), and headquarter (HQ) of the company as well as years of experience in the automotive industry (Y) in Table 1.

At the end of the interviews, we asked every interviewee about additional aspects of DL we had not discussed to collect data outside the three levels of DL. Moreover, we triangulated the interview data with publicly available data via an internet search and the companies' web presence.

Table 1. List of interviewees

\begin{tabular}{l|l|l|l|l}
\hline I & Department & ML & HQ & Y \\
\hline F1 & IT & F & GER & 9 \\
\hline F2 & Finance & F & GER & 14 \\
\hline F3 & IT & F & GER & 15 \\
\hline F4 & IT & F & GER & 14 \\
\hline
\end{tabular}

\begin{tabular}{l|l|l|l|l}
\hline F5 & R\&D & F & GER & 6 \\
\hline F6 & IT Strategy & F & GER & 23 \\
\hline F7 & Communication & F & GER & 9 \\
\hline F8 & Transformation & F & GER & 21 \\
\hline F9 & Strategy & F & GER & 3 \\
\hline F10 & Communication & F & GER & 6 \\
\hline F11 & Operations & F & AT & 2 \\
\hline L1 & Service & F & US & 21 \\
\hline L2 & Workers' council & Middle & FR & 24 \\
\hline L3 & Managing partner & Top & AT & 17 \\
\hline L4 & Digital Innovation & Low & GER & 18 \\
\hline L5 & Consulting & Top & GER & 20 \\
\hline L6 & Marketing & Low & IND & 9 \\
\hline L7 & Communication & Middle & FR & 11 \\
\hline L8 & HR & Top & UK & 12 \\
\hline L9 & HR & Middle & GER & 6 \\
\hline L10 & IT & Top & GER & 13 \\
\hline L11 & Digital Innovation & Top & GER & 8 \\
\hline L12 & Strategy & Top & GER & 24 \\
\hline L13 & Consulting & Middle & GER & 11 \\
\hline L14 & Marketing & Middle & GER & 19 \\
\hline
\end{tabular}

\subsection{Analysis}

To develop a coherent theory based on the collected data, we followed Corbin and Strauss' grounded theory [17]. First, the recorded data were transcribed, including grammatical and linguistical corrections, via the tool MaxQDA. Second, we performed an open, axial, and selective coding approach using English codes. Quotes were translated from German into English. The translation was reviewed by the authors. As there was no specific framework, we used open coding in the first step and coded the data regarding the digital leader, and the organizational and individual DL levels. Afterward, we used axial and selective coding to reveal relations and conflicts among codes leading to tensions and strategies in the FLR. We readjusted the interviews to specific areas of interest frequently mentioned by the interviewees. Then, we were able to build clusters based on the data and validate them with the remaining quarter of the interview candidates.

We considered and tried to minimize Maxwell's four types of validity threats [20] by taking the following countermeasures: (a) We collected "rich" data by giving interviewees time to speak without concerns and ask follow-up questions to reveal details of the situation. (b) We reduced bias and enriched data by triangulation with publicly available data. (c) Both authors carefully reviewed the research process and developed the results. 


\section{Findings}

To answer the question of causes of tensions in the FLR challenging the adoption of DL in the automotive industry and management strategies, we start with the understanding of DL in automotive practice. Then, we built four clusters based on the DLAS as evaluated by the followers and leaders to identify the causes of the tensions. In section 4.3 , the interviewees brought up four management strategies that we matched with the identified tensions.

\subsection{Understanding of DL and DLAS}

Analogous to the fuzziness about DL in existing literature, interviewees provided heterogenous explanations of DL independently of the company, department, or position. All interviewees stated that leading virtually and using digital technology to work together is part of DL. However, when we triangulated the definition of DL with the self-assessment of the DLAS (see figure 1), interviewees with lower DLAS scores described DL as transferring existing processes to digital tools without changes. In contrast, interviewees with higher DLAS scores emphasized the purpose of DL to build customer-focused digital BMs. Therefore, leaders need to enable people to be successful in digitalization and change the mindset and culture, and hierarchy of the company.

The companies of the interviewees show a broad variety in DLAS described as Learning to Walk to Gathering Speed as the average for most companies except the mobility startups. Individual teams within the corporation are characterized as Sprinters by offering digital products, whereas the majority is still generating ideas or transferring them from pilots to productions. "The gain in knowledge exists, but we are lacking the discipline to implement it globally" (F3). However, mobility startups and advanced teams within corporations offering digital BMs described organizational legitimacy building as their main challenge to further growth. Some interviewees reported that their company are in Ready to go as the digitalization of work is mainly driven by the Covid19 crisis but is not changing the way they work.

\subsection{Four configurations of FLR}

In the coding and triangulation process with publicly available data and literature, we observed and named four clusters of tensions in the FLR impeding the adoption of DL. The FLRs are clustered by their DLAS scores, so that quadrants show high similarity in (a) the followers' digital readiness and (b) DL adoption at the three levels of DL, e.g., F5 described the DLAS score as low as digitalization is only used for improving existing processes, so no adjustments take place on the leader, individual or organizational level and followers are lacking digital knowledge. Although the DLAS for leaders and followers is aligned in Left Behind and Volatile quadrants, tensions exist in the FLR of all quadrants.

In the Left Behind quadrant, low digital readiness matches low adoption of DL. The organizational level is optimized to produce physical products, and changes are incremental focusing on efficient production. Therefore, processes and procedures are digitalized, but not aligned to the digital attributes. Thus, the physical option is still preferred. Digital innovations are realized by strategic investments in startups with the intention of receiving the finalized, digital product. However, followers are not able to identify advantages in using technology for their work or the product portfolio but have concerns about data privacy and performance monitoring.

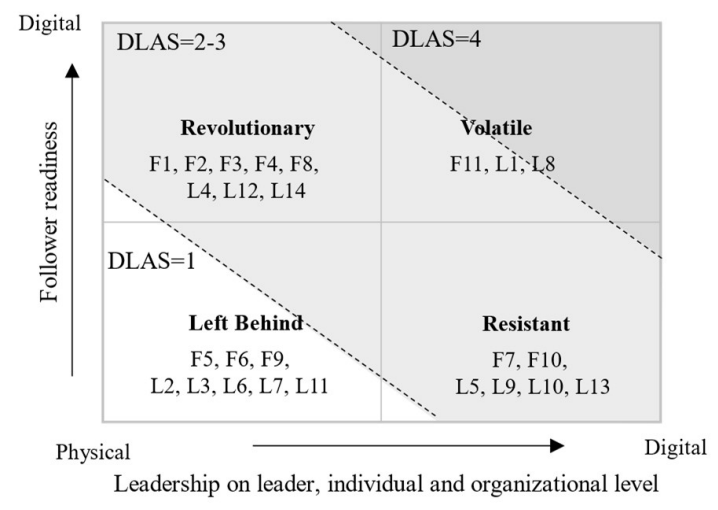

Figure 2. Clustering of FLR

In contrast to the Left Behind quadrant, the Revolutionary quadrant includes followers with high digital readiness resulting in intrinsic motivation to lead the change by bottom-up initiatives. The leadership is mainly aligned to the physical product. The DLAS level is Learning to Walk and Gathering Speed for the majority of the organization. Nevertheless, startups and incubators within these companies are characterized as Sprinters, as they are implemented to drive digital innovation but also spill over the digital organization to the core company. However, the challenge is the missing adaptability of the core company that is not able to take over the digital organization leading to followers' frustration.

The same heterogeneity of DLAS within one company exists in the Resistant quadrant, including companies that actively lead the change on the 
organizational and individual levels of leadership as "digitalization is here to stay" (F10). However, followers' digital awareness is low. Thus, they respond with skepticism about the uncertainty of the future and the frequency of the changes. Leaders listen to the concerns of followers and closely coach them in the transformation. In contrast, companies in the Volatile quadrant managed to set up a digital organization, focusing on the development of digital BMs. Therefore, followers are evaluated regarding their commitment, mindset, and capability of exploring new opportunities before being hired.

\subsection{Tensions between followers and leaders}

Based on the four quadrants, we investigated the causes of tensions in the FLR setting back the adoption of DL and categorized them by learning (LT), performing (PT), belonging (BT), organizing (OT) tensions as well as interrelations of the four tension categories (see section 2.2).

4.3.1. Left behind. Although in the FLR of the Left Behind quadrant followers and leadership are aligned on focusing on the physical BM, we identified the following tensions (see Table 2).

Table 2. Tensions in Left Behind

\begin{tabular}{l|l|l|l}
\hline \multicolumn{2}{|c}{ C } & Tension & LL \\
\hline T01 & PT::BT & $\begin{array}{l}\text { Lack of confidence in the } \\
\text { company's vision }\end{array}$ & O \\
\hline T02 & LT::PT & $\begin{array}{l}\text { Missing identification of } \\
\text { opportunities for the } \\
\text { individual }\end{array}$ & $\mathrm{I}$ \\
\hline T03 & OT & $\begin{array}{l}\text { Mismatch in people } \\
\text { management expectations }\end{array}$ & L \\
\hline C
\end{tabular}

$\mathrm{C}=$ Category, $\mathrm{LL}=$ Leadership Level, $\mathrm{O}=$

Organizational, $\mathrm{I}=$ Individual, $\mathrm{L}=$ Leader.

Tension exists in the lack of confidence regarding the company's vision. A leader stated, "We are successfully investing in partners to use their innovations for our core business" (L11). Followers observed that investments in the core business were canceled. However, in the past, the core business was celebrated as the main driver of success. Due to that, followers in the core business are struggling with the strategy of being a fast follower instead of a market leader (F5). Therefore, tensions arise from the individual's goal of belonging to the performing but not strategic collective (PT::BT).

Further tensions categorized as PT and LT exist as followers cannot identify opportunities in future BMs without impeding the existing performance. This lack of opportunities is based on three causes. First, followers felt insecure about their development because of previous failed attempts: "Three to five years ago, we built a vision, [...] had dreams, [...] and then we were disillusioned because it will never happen" (F6). Second, bottom-line orientation driven by Key Performance Indictors is part of the culture and rewards system in the automotive industry. Consequently, all investments must have a positive impact on the current BM. However, leaders are not confident that the impact can be realized. "It took us three to four years and a push by the Covid-19 crisis to decide that remote maintenance will save travel costs" (F6). Third, although agile methodologies and remote work are initiated by leadership at the organizational level, followers and leaders at the individual level do not adopt them by adjusting their work style. Therefore, "paper and pen will always be preferred" (F9). One example is the setup of virtual coffee breaks in remote work. A follower said, "We do not do virtual coffee meetings," (F5) and on the other side, leaders see the danger of "favoring people you know physically” (L11). Thus, work habits need to be adjusted to remote work.

In addition, OT results from a mismatch of people management expectations at the individual level of leadership. Followers and leaders are used to acting on a command-and-control basis. However, in DL followers ask for role models they can align to. " $A$ leader, who works analog, is not suitable for the job" (F9). In contrast, leaders are changing to support people by providing offerings, e.g., for learning. "We have a brought learning offering, but nobody is using it" (L11). Nevertheless, followers wait for the command to do something new, because otherwise they are not rewarded for it. Leaders are also not used to acting as a role model: "I spent less than $2 \%$ of my working hours for learning” (L11).

The tensions in FLR of the Left Behind quadrant lead to a lack of clarity about the digitalization target. Thus, followers and leaders do not know in which area they need to develop and have the fear that they are not part of the digital business.

4.3.2. Revolutionary. In the FLR of the Revolutionary quadrant, tensions are in areas that followers cannot change by themselves (see Table 3 ).

Table 3. Tensions in Revolutionary

\begin{tabular}{l|l|l|l}
\hline \multicolumn{2}{|c}{ C } & Tension & LL \\
\hline T04 & LT::OT & $\begin{array}{l}\text { Inadequacy of existing } \\
\text { organization, learning, } \\
\text { rewards processes for } \\
\text { digitalization }\end{array}$ & $\begin{array}{l}\text { O/ } \\
\text { I }\end{array}$ \\
\hline T05 & LT::BT & $\begin{array}{l}\text { Immutability of the } \\
\text { hierarchy }\end{array}$ & O \\
\hline
\end{tabular}




\begin{tabular}{l|l|l|l}
\hline T06 & BT & $\begin{array}{l}\text { Lack of appreciation for } \\
\text { leaders' qualifications }\end{array}$ & $\mathrm{L}$ \\
\hline $\mathrm{C}=$ Category, LL = Leadership Level, O $=$ \\
Organizational, I = Individual, L= Leader. \\
Followers in this quadrant are challenged by the
\end{tabular}
transfer of existing processes and policies at the organizational level to internal and external digital products. Thus, the tensions are characterized by renewing the organization to increase flexibility but still retaining stable organizational routines. Although some changes in the organization were initiated in favor of digitalization, such as the introduction of product owners, the traits and cognition of most people are still oriented to the physical environment. "The shell is new, but the inner is still the same" (F3). Especially, due to the increased number of policies caused by the emission violation scandal (also known as Dieselgate), employees feel they have no space to try out new things. "As leaders, we need to show the spot in the complex rule system within which people can act" (L4). However, people are tired of "fights between the physical and the digital world" (L14). Moreover, at the individual level, the highly standardized processes for learning and rewards do not match followers' demands (LT::OT). As both determinants are highly dependent on consultation with the workers' council, leaders mandate that followers use the process, even if it is not valuable. "It is a complete absurdity, [...] but everyone accepts it" (F1). Moreover, leaders report that they "only evaluate the results they can see" (L12). Therefore, followers starting initiatives are not rewarded for their behavior until the initiatives show results.

In addition, there is a tension characterized by learning and belonging (LT::BT), because followers drive change, but leaders emphasize existing power. Therefore, followers are highly engaged in co-creating and discussing a new culture and values by bottom-up activities, "you need to find a niche, where you are under-cover and then try out new things" (L4). However, the tensions are caused by the fact that bottom-up initiatives still need the "tone from the top" (F3) to have a global impact. This phenomenon is caused by the organization's deep hierarchical structure and thinking. Leaders emphasize that there must be a kind of hierarchy to organize a large cooperation. However, followers see the problem of using hierarchy and status symbols like corner offices and assistance for distancing and thus, retaining power. "Leaders' intent is to keep the existing, power and their ivory tower" (F3), and "constructive discourse is not rewarded" (F3). The impenetrability of the hierarchy combined with the dependency on the tone from the top makes it hard for bottom-up initiatives to succeed.
Furthermore, a belonging tension was identified based on the physical background of the leaders not matching the understanding of DL leading to mistrust of leaders' capabilities. In the past, "the best engineer" (F2), "the oldest in the team" (F4), was promoted to be a leader. "We do not develop leaders; we develop robots that are mini-mes" (F2). Thus, the high alignment to the hierarchy caused a lack of diversity in external automotive perspectives and skills in leadership positions. In addition, having a long career in the same department of the same company helped a leader in the hierarchy. However, in DL a leader needs to lead by purpose instead of tradition or hierarchy. Moreover, followers described the required leadership skills such as empathy, reflection, authenticity, visionary, and the mindset to try out and fail. Nevertheless, leaders still rest on the success of the past. "They speak condescendingly about the success of [competitors]" (F1) and try to avoid failures: "You don't want to fail" (L14).

The three tensions at the organizational/individual and leader levels lead to followers' and team leads' frustration, so that they stop driving the change of the organization and align with the antecedent organization. "You get tired of [tilting at] windmills", (L4) Moreover, followers think about leaving the company. "I think about quitting every two weeks to find an environment that better fits my agile values. However, change needs to be driven where it is needed the most" (F3).

4.3.3. Resistant. In the FLR of the Resistant quadrant, tensions are caused by the missing authentication of followers with DL at the organizational, and individual levels.

Table 4. Tensions in Resistant

\begin{tabular}{l|l|l|l}
\hline \multicolumn{2}{|l}{ C } & Tension & LL \\
\hline T07 & LT::OT & $\begin{array}{l}\text { Missing stability in new } \\
\text { openness of structures }\end{array}$ & O \\
\hline T08 & PT::BT & $\begin{array}{l}\text { Missing authentication } \\
\text { with leadership style }\end{array}$ & I \\
\hline T09 & BT & $\begin{array}{l}\text { Conflict of focusing on } \\
\text { individuals instead of } \\
\text { collectives }\end{array}$ & O \\
\hline C
\end{tabular}

$\overline{\mathrm{C}}=$ Category, $\mathrm{LL}=$ Leadership Level, $\mathrm{O}=$ Organizational, I = Individual.

Although the adoption of DL is high, and leaders managed to adjust processes and structures to drive digitalization, determinants such as culture or governance requiring a longer adoption period are not aligned to the new organization causing LT and OT. "We need to enable the core to be open for adopting to new circumstances" (L5). However, followers were 
socialist in a "fixed minded organization for ten, 20 or 30 years" (F7) providing stability and security. Therefore, people respond to the increased frequency of changes by escaping into routines and leftovers from the past, such as governance restrictions to exclude themselves as they are not able to translate the new organization to daily work: "Agile methods need to be well thought out before I can apply them" (F7).

The second tension (PT::BT) is caused by the application of a new leadership style by leaders aiming to transfer employees to the new BM, while followers focus on efficient performance. "In the past, we pushed towards efficiency, squeezed the lemon. Now we lead with the goal to replace $A$ [the traditional business] by B [the digital business]" (L5). Leaders moderate the transition by actively listening to followers' concerns. However, in a siloed organization, the follower depends highly on the direct leader regarding commands and career progress. Therefore, followers are not comfortable addressing concerns or critics to leaders, although leaders emphasize "if I say something wrong, you have to tell me" (L9). Tensions are caused by missing authentication of the follower with the new FLR.

In addition, followers identified BT regarding their own role within the collective of the company. In the past, followers' careers, learnings, tasks, and responsibilities were defined by group membership, for example, the department or production team. As DL aims for more flexibility to react to changes in digitalization, barriers based on hierarchies, structures, and processes are decreased, leading to more responsibility for followers to determine their professional activities. Therefore, followers need to be intrinsically motivated to work in cross-organizational communities. Moreover, career development has evolved: "The career path of employees was fixed; now, we start to enable professional careers" (L5). Followers need to choose their training opportunities. Therefore, tensions are caused because of integrating in the collective is replaced by followers taking over individual responsibilities outside the collective.

Because of the tensions in the FLR, the impact on followers is that they miss the opportunity to develop and be part of the transformation.

4.3.4. Volatile. In the FLR of the Volatile quadrant, the adoption of DL is challenged by tensions at the individual and organizational levels of leadership.

Table 5. Tensions in Volatile

\begin{tabular}{l|l|l|l}
\hline \multicolumn{2}{|c}{ C } & Tension & LL \\
\hline T10 & LT::PT & $\begin{array}{l}\text { Mismatch of changed } \\
\text { responsibilities and } \\
\text { accustomed work habits }\end{array}$ & I \\
\hline
\end{tabular}

\begin{tabular}{l|l|l|l}
\hline T11 & LT::OT & $\begin{array}{l}\text { Overwhelming evolvement } \\
\text { frequency of the } \\
\text { organization }\end{array}$ & $\mathrm{O}$ \\
\hline $\mathrm{C}=$ Category, $\mathrm{LL}=$ Leadership Level, $\mathrm{O}=$
\end{tabular}

Organizational, I = Individual.

First, LT and PT are caused by followers' changing responsibilities and constantly evolving work environment. As the organizational environment is developing leading-edge digital products, followers "need to think about solutions that do not exist at the moment" (F11). Thus, followers have more responsibility for developing and delivering results. Afterward, followers pitch ideas to boards to get decisions, although "people need to decide how to do it by themselves" (L1). One leader summarized the new responsibility as "everyone needs to be a leader" (L1). However, people with a corporate background are used to working in siloed and highly specialist positions. Thus, followers are not capable of overseeing and leading complex, cross-area topics. Moreover, followers' success is defined by standard and stretch goals. Because of the engineering culture of the past, followers are not comfortable with not achieving stretch goals with a perfectly engineered solution. In addition, followers are challenged by the ambiguity of the environment causing frequent changes, which also contradict the followers' results. "You need to get comfortable with throwing away $50 \%$ of your work" (F11). Leaders try to mitigate this tension by increasing clarity about this work environment in the hiring process: "People need to be committed to this risk" (L8). However, a leader also admitted that the process of paying attention to followers' commitment needs to be improved.

Second, LT and OT are caused by the emergence of the organization while followers demand organizational stability. "We don't know where we go, but this is our vision for the world" (L1). Therefore, constant changes are made in the strategy, values, and processes. "Our organization is like a skeleton; the inside is very fluid and flexible" (L8). The fluidity is caused by the demand to build organizational legitimacy. "Our whole startup is a learning experience for people [...] as we are questioning the whole ecosystem and we work in a digital nomad organization" (L8). Although followers identify with the company's vision and values, they explain the challenge "to connect to the company's spirit" (F11) especially in remote work. Moreover, one follower described the challenge of keeping the high ambiguity in the company structure as a key differentiator from large corporations: "We need the discipline to keep decisions locally and maintain flat hierarchies when the company grows" (F11). 
These tensions in the FLR cause employees to highly commit to their work or quit. "One follower told me: You can call me again if you have settled, but this is not my world" (L8).

\subsection{Strategies for managing tensions}

Besides providing the first investigation of tensions in the FLR in the adoption of DL above, the interviewees suggested in total four different strategies for managing these tensions that are discussed, planned, piloted, or implemented in practice. In the following, we match the tensions with the strategies.

The first strategy is up- and re-skilling (S1) of followers and leaders to increase transparency and adoption of digitalization. Aiming for upskilling, DL trainings for leaders as well as technological and methodological trainings for all employees are offered. Re-skilling is driven by developing horizontal career path and development plans, as well as programs like programming school. This strategy helps followers develop in alignment with the vision (T01) and prepare for new responsibilities (T10).

The second strategy is focusing on transparency and sustainable implementation of DL (S2). Increasing transparency is driven by discussions with followers. Thus, the adoption process of DL as well as the impact on the company performance and the individual is clarified (T01, T02). Moreover, changes at the DL organizational level are sustainably implemented by leveraging rewards and penalties. A follower reported that "we point out if someone is not acting according to our values" (F1). Peer group feedback rewards followers for acting aligned to vision and values. Leaders also emphasize that "people that are toxic to the change need to be removed" (L5). Applying this strategy mitigates the tensions of resistance in the hierarchy (T05) and organization (T04).

To help followers during the transition, the strategy is to adjust the leadership style (S3) according to the conditions of the followers' work situation. Therefore, "if you work in production you need to stick to the processes. However, when there is an interruption, you need to switch and work agilely together in swarms" (L9). In addition, in the transition, followers, depending on their progress in the digital readiness, require a different leadership style, for example, being a role model, empowering, or giving commands to synchronize the leader's style and the followers' demand (T03, T08, and T11).

Last, leaders increase the cognitive diversity (S4) of all employees. By organizing Bar camps, providing think tanks or exchange programs to startups or Silicon Valley, leaders motivate followers to get new perspectives and participate in discussions about rethinking the existing. The goal is that followers and leaders can flexibly adjust and understand the characteristics of both sides. The strategy solves the tension of qualifying leaders for DL (T06), followers' comfort with constant changes (T07, T11), and frequent integration in different groups (T09).

\section{Discussion}

We investigated the tensions in the FLR challenging the adoption of DL. Leveraging the automotive industry as an industry in the middle of the transition, we conducted 25 expert interviews with followers and leaders in the automotive industry, and using grounded theory, we identified (a) 11 tensions based on Smith and Lewis [8] clustered by DLAS and (b) four strategies for managing these tensions. In this section, we discuss the findings with respect to the literature on DL.

First, we contribute to DL research by adding the perspective of the follower. Thus, we provide the first investigation of tensions in the FLR in the adoption of DL in the automotive industry. Although Bolte et al. [6] focused on the attributes of leadership in digitalization from a follower and leader perspective, our findings show tensions impeding the adoption of DL. In the automotive industry, the followers are the main population that DL needs to address. We identify the tensions at the organizational, individual, and leader levels.

Second, the findings enrich research on DL adoption with strategies for managing the tensions in the FLR. In addition to learning, transparency, and sustainable implementation, as well as cognitive diversity, the findings emphasize the importance of adjusting the leader's style to the follower's digital readiness. Until now, the DL leadership style had rarely been analyzed. Prince [12] concluded that DL leverages authentic, transformational, and transactional leadership styles. However, Prince missed the condition in which they are applied.

The findings are relevant for practitioners and academics. First, leaders and followers in practice facing challenges in adopting DL can identify their own position in the FLR cluster and find strategies for managing their tensions. Second, we close the research gap of (a) the missing follower perspective by including followers to uncover 11 tensions in the FLR and (b) overcome the tensions in the DL adoption process by identifying four strategies for managing tensions in the automotive industry aiming for digital BMs.

The limitations of this paper are twofold. First, we focused on tensions in the FLR and excluded 
interorganizational tensions between the organization and the ecosystem of suppliers and customers.

Second, there are four types of limitations according to Maxwell [20] resulting from the qualitative research design. The first threat is evaluative, impacting validity because of the individual interpretation of the data. Although we triangulated data with literature and publicly available data, the threat cannot be completely mitigated. The second threat is theoretical, resulting from previous knowledge about DL research, although we conducted interviews and coding with an open mindset. The third threat is interpretative, resulting from the subjectivity of both authors. We took mitigation actions as both authors carefully reviewed the research process and results. Moreover, the interview guide was readjusted to validate the interpretation of the results. The fourth threat impacts the study's validity because of the description process. To mitigate, the results were interpreted iteratively by both authors and validated by the last third of the interviewees.

\section{Conclusion and outlook}

The automotive industry is "off to new pastures"; however, it is struggling with detaching from the ancient pastures. This struggle leads to high heterogeneity of followers and leaders adopting DL, as the physical BM is still relevant in their work environment. This investigation improved our understanding of tensions in the FLR impeding the adoption of DL, as well as strategies for managing these tensions based on 25 perspectives of followers and leaders from different automotive companies.

Additional research is required to further (a) understand DL, especially leadership styles, (b) differentiate dilemma and paradox tensions, and (c) investigate in more depth the adoption process of DL. In the future, our work will aim for a better understanding of DL and its adoption.

\section{References}

[1] E. Piccinini, A. Hanelt and R. W. Gregory, "Transforming Industrial Business: The Impact of Digital Transformation on Automotive Organizations", 36th International Conference on Information Systems, ICIS, AIS, Fort Worth, 2015.

[2] B. Hildebrandt, A. Hanelt and S. Firk, "Entering the Digital Era - The Impact of Digital Technology-related M\&As on Business Model Innovations of Automobile OEMs", 36th International Conference on Information Systems, ICIS, AIS, Fort Worth, 2015.

[3] Volkswagen AG, "Dr. Herbert Diess - Excerpts from his speech at the GLOBAL Top Management Conference", https://www.volkswagenag.com/presence/konzern/doc uments/Herbert_Diess_GTMC_2019_EN.pdf (Accessed: 2021/04/18).

[4] J. Van Ee, I. El Attoti, P. Ravesteyn, and B. M. E. De Waal, "BPM Maturity and Digital Leadership: An exploratory study", Communications of the IIMA, $18(1), 2020$.

[5] J. K. Eberl, and P. Drews, "Digital Leadership Mountain or Molehill? A Literature Review", 16th International Conference on Wirtschaftsinformatik, AIS, Duisburg \& Essen, 2021.

[6] S. Bolte, J. Dehmer, and J. Niemann, "Digital Leadership 4.0", Acta Technica Napocensis, 2018, pp. 637-646.

[7] B. J. Avolio, F. O. Walumbwa, and T. J. Weber, "Leadership: current theories, research, and future directions", Annual review of psychology, 2009, pp. 421-449.

[8] W. K. Smith, and M. W. Lewis, "Toward a Theory of Paradox: A dynamic Equilibrium Model of organizing", Academy of Management Review, 36(2), 2011, pp. 381-403.

[9] M. F. Klus, J. Müller, Identifying leadership skills required in the digital age, Westfälische WilhelmsUniversität, Münster, 2018.

[10] B. J. Avolio, S. Kahai and G. E. Dodge, "ELeadership: Implications for Theory, Research and Practice", Leadersh. Q., 2001, pp. 615-668.

[11] O. A. El Sawy, P. Kræmmergaard, H. Amsinck, and A. Lerbeck Vinther, "How LEGO Built the Foundations and Enterprise Capabilities for Digital Leadership", MIS Q. Executive, 2016, pp. 141-166.

[12] K. A. Prince, Digital leadership: transitioning into the digital age, James Cook University, Queensland, 2018.

[13] T. Schwarzmüller, P. Brosi, D. Duman, and I. M. Welpe, "How Does the Digital Transformation Affect Organizations? Key Themes of Change in Work Design and Leadership", Management Revue, 29(2), 2018, pp. 113-137.

[14] P. Weill, S. L. Wörner, and F. González, "Is Your Company a Digital Leader or a Digital Laggard?", MIT Sloan CISR, XVII(3), 2017, pp. 1-4.

[15] S. Chanias and T. Hess, ed., Understanding Digital Transformation Strategy Formation: Insights from Europe's Automotive industry, 2016.

[16] M. W. Lewis, "Exploring Paradox: Toward a More Comprehensive Guide", The Academy of Management Review, 25(4), 2000, p. 760.

[17] J. M. Corbin, and A. Strauss, "Grounded Theory Research: Procedures, Canons, and Evaluative Criteria", Qualitative Sociology, 1990, pp. 3-21.

[18] M. D. Myers, and M. Newman, "The Qualitative Interview in IS Research: Examining the Craft", Information and Organization, 2007, pp. 2-26.

[19] Adapt2Digital Ltd., "Organisation Transformation Assessment", https://www.adapt2digital.com/digitalmaturity-assessment-1 (Accessed: 2021/05/01).

[20] J. A. Maxwell, Qualitative Research Design: An Interactive Approach, SAGE, Washington, 2013. 